\title{
Asymptotic Behaviour of Particle Motion Under Repulsive Forces
}

\author{
G. Galperin* \\ Department of Mathematics, University of Maryland, College Park, MD 20742, USA
}

\begin{abstract}
The asymptotic nature of motions, as time tends to infinity, is investigated for classical point particles interacting by repulsive two-body potentials $U_{i j}$. It is found that the conditions $\int_{1}^{\infty} U_{i j}(r) d r<\infty$ are necessary and sufficient for asymptotically straight line uniform motion. In the case of equal asymptotic velocities the proof depends only on a certain property of the motion ("partial center of mass convexity") implied by the repulsivity of the potentials.
\end{abstract}

\section{Introduction and Results}

This paper continues the previous work of the author $[1,7]$ and that of Vaserstein [8], as well as Moser [2], Himchenko and Sinai [6] (see also related works of Sinai $[4,5])$. Moser $[2]$ considered a system of two particles moving along a line under the action of a strictly repulsive potential $U$, and he analyzed the connection between the asymptotic properties of the motion in the limit as the time $t \rightarrow \infty$, and the potential $U$. Under certain conditions imposed on $U$ and also supposing that the asymptotic motion is of the form $v_{\infty} t+c+O(1)$ as $t \rightarrow \infty$, an explicit formula for this connection was obtained. In [6] the concept of a reflectionless potential is introduced and investigated. This means that for a system of particles moving on a line their motion is asymptotically uniform, i.e., of the form $v_{\infty} t+c+o(1)$, both when $t \rightarrow+\infty$ and when $t \rightarrow-\infty$, and the set of asymptotic velocities is the same in the two limits $t \rightarrow \pm \infty$, even though the asymptotic velocity of any one particular particle can be different at $-\infty$ and $+\infty$. The theorem is proved that any potential $U(r)$ which satisifies $U(r) \sim C_{0} r^{-\alpha}, U^{\prime}(r) \sim C_{1} r^{-\alpha-1}, U^{\prime \prime}(r) \sim C_{2} r^{-\alpha-2}$ as $r \rightarrow \infty$ for some $\alpha>2$, cannot be reflectionless.

\footnotetext{
* The original version of this paper was edited and re-written by A. Lenard from Indiana University. The author expresses his gratitude to Professor Lenard for his work which has significantly improved both the mathematical presentation and the style of the paper
} 
For a general system of point particles moving in $N$-dimensional space, and described by the position vectors $\mathbf{r}_{1}(t), \mathbf{r}_{2}(t), \ldots, \mathbf{r}_{n}(t)$, we say that the motion is asymptotically uniform in the limit $t \rightarrow+\infty$ if vectors $\mathbf{w}_{i}, \mathbf{c}_{i}$ exist such that

$$
\lim _{t \rightarrow+\infty} \frac{d \mathbf{r}_{i}}{d t}=\mathbf{w}_{i}
$$

and

$$
\lim _{t \rightarrow+\infty}\left(\mathbf{r}_{i}-t \mathbf{w}_{i}-\mathbf{c}_{i}\right)=0
$$

for $i=1,2, \ldots, n$, conditions briefly written $\mathbf{v}_{i}(t)=\mathbf{w}_{i}+o(1)$ and $\mathbf{r}_{i}(t)=t \mathbf{w}_{i}+\mathbf{c}_{i}+o(1)$ as $t \rightarrow+\infty$. The $\mathbf{w}_{i}$ are called the asymptotic velocities and the $\mathbf{c}_{i}$ the asymptotic phases. The existence of the asymptotic velocities is insufficient, in general, to assure the existence of the asymptotic phases. A simple example illustrates this. Let $n=2$, and let us discuss the motion of two particles in terms of the relative separation $\mathbf{r}(t)=\mathbf{r}_{1}(t)-\mathbf{r}_{2}(t)$, the reduced mass $m=m_{1} m_{2} /\left(m_{1}+m_{2}\right)$, and the potential taken to be $U=U(|\mathbf{r}|)=U(r)=r^{-1}$. For purely radial motion the differential equation

$$
m \frac{d^{2} r}{d t^{2}}=\frac{1}{r^{2}}
$$

possesses the energy constant $E$, and one finds

$$
\begin{aligned}
t & =\int_{r_{0}}^{r}\left(\frac{2 E}{m}-\frac{2}{m s}\right)^{-1 / 2} d s=\left(\frac{m}{2 E}\right)^{1 / 2} \int_{r_{0}}^{r}\left(1+\frac{1}{2 E s}+O\left(\frac{1}{s^{2}}\right)\right) d s \\
& =\left(\frac{m}{2 E}\right)^{1 / 2}\left[r+\frac{1}{2 E} \log r+O(1)\right]
\end{aligned}
$$

as $t$ and $r \rightarrow+\infty$, and this is incompatible with an asymptotic behaviour of the form $r(t)=a t+b+o(1)$ as $t \rightarrow+\infty$.

No previous study examined the conditions under which asymptotic motion is in fact uniform. In the present paper we offer such a condition for repulsive potentials. Roughly speaking, it is necessary and sufficient that the integral of the potential function, extended over the radial variable shall be convergent (for a precise statement see below). We consider $n$ point particles of masses $m_{i}$, position vectors $\mathbf{r}_{i}(t)$, and denote the force acting on the particle $i$ by $\mathbf{F}_{i}$. We assume the forces to be of the two-body type, i.e. $\mathbf{F}_{i}=\sum_{j \neq i} \mathbf{F}_{i j}$, where $\mathbf{F}_{i j}=-\mathbf{F}_{j i}$ is the force on particle $i$ due to the presence of particle $j$. Conservative forces are assumed, so that $-\mathbf{F}_{i j}$ is the gradient with respect to the variable $\mathbf{r}_{i}$ of a potential function $U_{i j}$ depending only on the separation $r_{i j}=\left|\mathbf{r}_{i}-\mathbf{r}_{j}\right|$. Finally, we assume repulsive forces: Each $U_{i j}(r)$ is a non-increasing function of $r$ and $U_{i j}(\infty)=0$. We make the technical assumption that the $U_{i j}$ have continuous derivatives $U_{i j}^{\prime}$. About the behaviour for $r \rightarrow 0$ we assume either that $U_{i j}(r) \rightarrow+\infty$, in which case $r_{i j}$ remains bounded away from zero for any motion, or else if $U_{i j}(0)<\infty$ we assume $U_{i j}^{\prime}(0)=0$ so that the equations of motion are unambiguous even when the coordinates $\mathbf{r}_{i}$ and $\mathbf{r}_{j}$ coincide.

Our first result is simple, and we give its proof below. 
Theorem 1. Assuming the hypotheses above, if all possible motions in the two-body problem are asymptotically uniform in the limit $t \rightarrow \infty$ then $\int_{1}^{\infty} U(r) d r<\infty$.

Proof. We examine radial outward motion for the two-particle system for $t \geqq 0$. Let $r=\left|\mathbf{r}_{1}-\mathbf{r}_{2}\right|, v=\frac{d r}{d t}, m$ the reduced mass and $U=U_{R}(r)$ the potential. Let $a=r(0)>0$, so that $v(0)>0$ and $v(t)$ as well as $r(t)$ are increasing functions for $t>0$. By assumption $w=\lim _{t \rightarrow \infty} v(t)$ and $\lim _{t \rightarrow \infty}[r(t)-t w]$ exist. Let $T>0$ and $R=r(T)$. Because of the conservation of energy $U(r)=\frac{m}{2} w^{2}-\frac{m}{2} v(t)^{2}$ we may change variable in the integral and write

$$
\int_{a}^{R} U(r) d r=\frac{m}{2} \int_{0}^{T}\left[w^{2}-v(t)^{2}\right] v(t) d t
$$

Since $v(t) \leqq w$ for all $t \geqq 0$, we obtain the inequality

$$
\begin{aligned}
\int_{a}^{R} U(r) d r & \leqq m w^{2} \int_{0}^{T}[w-v(t)] d t \\
& =m w^{2}(T w+a-R) .
\end{aligned}
$$

But the right hand side has a limit as $T$ and, with it, $R \rightarrow \infty$, so it remains and the desired conclusion follows.

Our second result is more complicated and requires a few words of explanation. We recall that in Vaserstein's paper [8] and in the author's paper [7] it was shown that from the repulsivity of the potential it follows that asymptotic velocities exist. In fact, this conclusion depends only on a very general "repulsivity condition" which does not require for its formulation a conservative dynamical system and, indeed, not even Newtonian equations of motion. At any rate, here we can take the existence of the asymptotic velocities for granted and the task is to show that, under suitable conditions, the asymptotic phases (see above) also exist, i.e., the motion is asymptotically uniform. This condition is suggested by Theorem 1, namely the convergence of all the integrals $\int_{1}^{\infty} U_{i j}(r) d r$. Since this involves only the behaviour of the two-body potentials at large separation $r_{i j} \rightarrow \infty$, it is natural that the desired conclusion is easiest reached if we restrict ourselves to motions for which all interparticle separations $r_{i j} \rightarrow \infty$ as $t \rightarrow+\infty$. This will be the case if the $n$ asymptotic velocities $\mathbf{w}_{i}$ are all distinct, a type of motion which, for obvious reasons, we shall call generic. A type of motion which is not generic will be called exceptional, thus in the exceptional case $\mathbf{w}_{i}=\mathbf{w}_{j}$ for at least one pair $i \neq j$.

Theorem 2. Assuming the hypotheses above, if $\int_{1}^{\infty} U_{i j}(r) d r<\infty$ for $1 \leqq i<j \leqq n$, then all possible generic motions for the n-particle system are asymptotically uniform in the limits $t \rightarrow \pm \infty$. 
The proof will be given in Sect. 2 of this paper. The main idea is that on account of $\mathbf{w}_{i} \neq \mathbf{w}_{j}$ the separation $r_{i j}(t)$ grows roughly linearly with time, and this makes possible sufficiently good estimates on certain integrals to assure their finiteness. From these in turn one demonstrates that the Cauchy condition holds for the functions $\mathbf{r}_{i}(t)-t \mathbf{w}_{i}$ in the limit $t \rightarrow \infty$ yielding the asymptotic phases $\mathbf{c}_{i}$.

The further question arises about the exceptional motions. In such a motion some of the $r_{i j}$ remain bounded, and the corresponding particles have, so to speak, exactly the right amount of energy to "run up a potential hill" and come to rest where the potential has vanishing gradient. This is analogous to the behaviour of a pendulum so set in motion that it comes to a stop exactly at the upper unstable equilibrium position. To deal with this case ideas quite different from the generic case are required. We do not even need to use the differential equations of motion, only a particular property of motions implied by the repulsivity of the forces. This property may be stated as follows: Let $p$ be a hyper plane in $\mathrm{N}$-dimensional space, and let $d=d(t)$ denote the distance of the center of mass, from $p$, of those particles that are at time $t$ strictly inside one of the half-spaces determined by $p$. Then for any $\tau$ there is an interval $\tau-\varepsilon<t<\tau+\varepsilon$ such that in it $d(t)$ is a convex function. Indeed, for the interval we may take $\varepsilon$ so small that the chosen particles remain separated from the others by a plane parallel to $p$. Take a coordinate axis perpendicular to $p$ and positive towards the chosen half-space. Then $d(t)$ is the coordinate of the center of mass of the chosen particles. It satisfies the differential equation $M d^{\prime \prime}(t)$ $=F(t)$, where $M$ is the total mass of the particles in the chosen half-space and $F(t)$ is the component of the total force acting on those particles. But $F(t)$ is the sum of terms, all of them non-negative, since interparticle forces between particles on different sides of the separating plane are only relevant, and the components of these the coordinate axis are non-negative (repulsivity). Let us call the property just enunciated PCMC ("partial center of mass convexity"). In Sect. 3 the following will be proved.

Theorem 3. Suppose that a motion has PCMC, and suppose that all asymptotic velocities exist and are equal. Then all asymptotic phases exist as well.

\section{Proof of Theorem 2}

The Newtonian equations of motion are

$$
m_{i} \frac{d^{2} \mathbf{r}_{i}}{d t^{2}}=-\sum_{j \neq i} U_{i j}^{\prime}\left(r_{i j}\right) \boldsymbol{\omega}_{i j},
$$

where $\boldsymbol{\omega}_{i j}$ is the vector of unit length in the direction of $\mathbf{r}_{i}-\mathbf{r}_{j}$. Incorporating the initial conditions

$$
\left\{\begin{aligned}
\mathbf{r}_{i}(0) & =\mathbf{a}_{i} \\
\mathbf{v}_{i}(0) & =\mathbf{b}_{i}
\end{aligned}\right.
$$

we integrate (2.1) twice

$$
\mathbf{r}_{i}(t)=\mathbf{a}_{i}+t \mathbf{b}_{i}+\frac{1}{m_{i}} \sum_{j \neq 1}\left[t \mathbf{A}_{i j}(t)-\mathbf{B}_{i j}(t)\right]
$$


where

$$
\mathbf{A}_{i j}(t)=-\int_{0}^{t} U_{i j}^{\prime}\left(r_{i j}(\tau)\right) \boldsymbol{\omega}_{i j}(\tau) d \tau
$$

and

$$
\mathbf{B}_{i j}(t)=-\int_{0}^{t} \tau U_{i j}^{\prime}\left(r_{i j}(\tau)\right) \boldsymbol{\omega}_{i j}(\tau) d \tau
$$

Lemma 1. If the derivative of a vector valued function $\mathbf{r}(t)$ has a non-zero limit as $t \rightarrow+\infty$, then the derivative of its absolute value is bounded away from zero on some interval $[T, \infty)$.

Proof. Let $\mathbf{w}=\lim _{t \rightarrow+\infty} \frac{d \mathbf{r}}{d t}$, then $\mathbf{r}(t)=\mathbf{r}(0)+t \mathbf{w}+\int_{0}^{t}\left(\frac{d \mathbf{r}(\tau)}{d \tau}-\mathbf{w}\right) d \tau$ shows that $\lim _{t \rightarrow+\infty} \frac{\mathbf{r}(t)}{|\mathbf{r}(t)|}=\frac{\mathbf{w}}{|\mathbf{w}|}, \quad$ and $\quad$ therefore $\quad \lim _{t \rightarrow+\infty} \frac{d}{d t}|\mathbf{r}(t)|=\lim _{t \rightarrow+\infty} \frac{\mathbf{r}(t)}{|\mathbf{r}(t)|} \cdot \frac{d \mathbf{r}(t)}{d t}=|\mathbf{w}|>0$.

Since it follows from our hypotheses (see $[7,8]$ ) that the particles have asymptotic velocities, and we are considering the generic case, we may apply Lemma 1 to the function $\mathbf{r}_{i}(t)-\mathbf{r}_{j}(t)$, and conclude that

$$
\frac{d r_{i j}(t)}{d t} \geqq \alpha>0
$$

for some constant $\alpha$ and all sufficiently large $t$ which, without restriction of generality, we may take $t \geqq 0$. Let $0 \leqq t_{1}<t_{2}$, and consider the difference of two values of (2.4) for $t=t_{2}$ and $t=t_{1}$. By changing variable of integration, noting (2.6) and the fact that $U_{i j}^{\prime} \leqq 0$, we obtain the estimate

$$
\begin{aligned}
\left|\mathbf{A}_{i j}\left(t_{2}\right)-\mathbf{A}_{i j}\left(t_{1}\right)\right| & \leqq-\frac{1}{\alpha} \int_{a_{1}}^{a_{2}} U_{i j}^{\prime}(r) d r \\
& \leqq \frac{1}{\alpha} U_{i j}\left(a_{1}\right),
\end{aligned}
$$

where $a_{1}=r_{i j}\left(t_{1}\right), a_{2}=r_{i j}\left(t_{2}\right)$. As $t_{1} \rightarrow+\infty$ the right hand side has the limit zero, and so by the Cauchy convergence criterion

$$
\mathbf{A}_{i j}(\infty)=\lim _{t \rightarrow \infty} \mathbf{A}_{i j}(t)
$$

exists. Now replace $t_{1}$ by $t$ in (2.7) and let $t_{2} \rightarrow+\infty$. Since $r_{i j}(t) \geqq \alpha t+\beta$ with $\beta=r_{i j}(0)$ on account of $(2.6)$, we obtain

$$
\left|\mathbf{A}_{i j}(t)-\mathbf{A}_{i j}(\infty)\right| \leqq \frac{1}{\alpha} U_{i j}(\alpha t+\beta)
$$

Lemma 2. Suppose $f(r)$ is a non-increasing non-negative function such that $\int^{\infty} f(r) d r<\infty$. Then $f(r)=o\left(\frac{1}{r}\right)$ as $r \rightarrow+\infty$. 
Proof. Let $\varepsilon>0$ be arbitrary and $R$ so large that $\int_{R}^{\infty} f(r) d r \leqq \varepsilon$. If $x \geqq 2 R$ then $\varepsilon \geqq \int_{R}^{\infty} f(r) d r \geqq \int_{R}^{x} f(r) d r \geqq(x-R) f(x) \geqq \frac{1}{2} x f(x)$. This shows $\lim _{x \rightarrow+\infty} x f(x)=0$, as required.

Applying Lemma 2 to (2.9) we now have

$$
\mathbf{A}_{i j}(t)=\mathbf{A}_{i j}(\infty)+o\left(\frac{1}{t}\right)
$$

as $t \rightarrow+\infty$. The integrals (2.5) are treated similarly, the extra factor $\tau$ being bounded from above by $\frac{1}{\alpha}\left(r_{i j}-\beta\right)$. Thus for $0 \leqq t_{1}<t_{2}$

$$
\begin{aligned}
\left|\mathbf{B}_{i j}\left(t_{2}\right)-\mathbf{B}_{i j}\left(t_{1}\right)\right| & \leqq-\frac{1}{\alpha^{2}} \int_{a_{1}}^{a_{2}}(r-\beta) U_{i j}^{\prime}(r) d r \\
& =\frac{1}{\alpha^{2}}\left\{\left(a_{1}-\beta\right) U_{i j}\left(a_{1}\right)-\left(a_{2}-\beta\right) U_{i j}\left(a_{2}\right)+\int_{a_{1}}^{a_{2}} U_{i j}(r) d r\right\} .
\end{aligned}
$$

As $t_{1} \rightarrow+\infty$ we have $a_{1} \rightarrow+\infty$ and $a_{2} \rightarrow+\infty$, and since $\int^{\infty} U_{i j}(r) d r<\infty$ and Lemma 2 applies, the right hand side of (2.11) tends to zero. This shows that

$$
\mathbf{B}_{i j}(\infty)=\lim _{t \rightarrow+\infty} \mathbf{B}_{i j}(t)
$$

exists. Substitute (2.10) into (2.3) and make use of (2.12). This yields

$$
\mathbf{r}_{i}(t)=t \mathbf{w}_{i}+\mathbf{c}_{i}+o(1),
$$

where

$$
\mathbf{w}_{i}=\mathbf{b}_{i}+\frac{1}{m_{i}} \sum_{j \neq i} \mathbf{A}_{i j}(\infty)
$$

and

$$
\mathbf{c}_{i}=\mathbf{a}_{i}-\frac{1}{m_{i}} \sum_{j \neq i} \mathbf{B}_{i j}(\infty)
$$

Furthermore, upon differentiating (2.3) one obtains

$$
\mathbf{v}_{i}(t)=\frac{d \mathbf{r}_{i}(t)}{d t}=\mathbf{b}_{i}+\frac{1}{m_{i}} \sum_{j \neq i} \mathbf{A}_{i j}(t)
$$

This shows that the

$$
\mathbf{w}_{i}=\lim _{t \rightarrow+\infty} \mathbf{v}_{i}(t)
$$

are the asymptotic velocities, and the $\mathbf{c}_{i}$ the asymptotic phases.

This completes the proof of Theorem 2 . 


\section{Proof of Theorem 3}

By making use of Gallilean invariance of the mechanical problem, it is no restriction of generality to assume, as we shall, that the asymptotic velocities are zero. Therefore we must prove in this case that the limits

$$
\mathbf{c}_{i}=\lim _{t \rightarrow+\infty} \mathbf{r}_{i}(t)
$$

exist. We choose an arbitrary directed line as a coordinate axis, denoting the component of $\mathbf{r}_{i}(t)$ along it by $x_{i}(t)$, so that the existence of $\lim x_{i}(t)$ as $t \rightarrow+\infty$ has to be shown.

We shall adopt the convention that the $\xi_{i}(t)(i=1,2, \ldots, n)$ are the $x_{i}(t)$ arranged in increasing numerical order

$$
\xi_{1}(t) \leqq \xi_{2}(t) \leqq \ldots \leqq \xi_{n}(t),
$$

and for the whole configuration we just write $\xi(t)$ without a subscript. We also use $\xi=\left(\xi_{1}, \xi_{2}, \ldots, \xi_{n}\right)$ as a symbol for a general ordered configuration independently of any motion. The masses $m_{i}$ are associated with the coordinates $x_{i}$, and let $\mu_{i}$ be the rearrangement of the $m_{i}$ such that $\mu_{i}$ corresponds to the position $\xi_{i}$. $m$ or $\mu$ without subscript refers to the assembly of all masses.

For any system $\mu=\left(\mu_{1}, \mu_{2}, \ldots, \mu_{n}\right)$ of masses and corresponding ordered positions $\xi=\left(\xi_{1} \leqq \xi_{2} \leqq \ldots \leqq \xi_{n}\right)$, we define a function $f(\sigma)$ for $\sigma>0$ as follows

$$
f(\sigma)= \begin{cases}\xi_{1} & 0<\sigma \leqq \mu_{1}, \\ \xi_{2} & \mu_{1}<\sigma \leqq \mu_{1}+\mu_{2}, \\ \xi_{3} & \mu_{1}+\mu_{2}<\sigma \leqq \mu_{1}+\mu_{2}+\mu_{3}, \\ \text { etc. } & \end{cases}
$$

and define

$$
F(\sigma)=\int_{0}^{\sigma} f\left(r^{\prime}\right) d r^{\prime} .
$$

Thus if we write $M_{j}=\mu_{i}+\ldots+\mu_{j}$ we have

$$
F\left(M_{j}\right)=\mu_{1} \xi_{1}+\ldots+\mu_{j} \xi_{j},
$$

and for other arguments $F$ is defined by piecewise linear interpolation. When we consider motions $x(t)$ and the corresponding ordered configurations $\xi(t)$ we shall write $f_{t}(\sigma)$ respectively $F_{t}(\sigma)$ to indicate the time dependence. Note that (3.4) is a positive multiple by the factor $M_{j}$ of the center of mass coordinate of the $j$ left-most particles in the configuration. Thus if the motion has the PCMC property (see Sect. 1) then from $\xi_{j}\left(t_{0}\right)<\xi_{j+1}\left(t_{0}\right)$ we can conclude that $F_{t}\left(M_{j}\right)$ is a concave function of $t$ for some interval surrounding $t_{0}$, indeed for an interval throughout which the strict inequality $\xi_{j}(t)<\xi_{j+1}(t)$ holds.

Lemma 3. Suppose $x(t)$ is a motion having the PCMC property. Then $F_{t}(\sigma)$ is a concave function of $t$ for all $\sigma>0$.

Before proving this we make some preliminary remarks. 
A function is concave if and only if through any point on its graph one can pass a line segment such that in some neighborhood of the point the graph of the function does not rise above the segment. We shall use this to show that a function $\phi(t)$ is concave by the following device: For each $t_{0}$ we exhibit a function $\psi(t)$, defined and concave in some neighborhood of $t_{0}$, and such that $\phi\left(t_{0}\right)=\psi\left(t_{0}\right)$ and $\phi(t) \leqq \psi(t)$ for $t$ in that neighborhood.

We shall use a simple inequality obtained as follws. Let $\phi(s)$ be non-decreasing and $\Phi(s)=\int_{0}^{s} \phi^{\prime}\left(s^{\prime}\right) d s^{\prime}$. Let $a<c$ and $a \leqq b \leqq c$. Then

$$
\begin{aligned}
\Phi(b) & =\Phi(a)+\int_{a}^{b} \phi^{\prime}(s) d s \\
& \leqq \Phi(a)+(b-a) \phi(b),
\end{aligned}
$$

and similarly

$$
\Phi(b) \leqq \Phi(c)-(c-b) \phi(b)
$$

Taking the appropriate convex combination of these inequalities we obtain

$$
\Phi(b) \leqq \frac{c-b}{c-a} \Phi(a)+\frac{b-a}{c-a} \Phi(c) .
$$

The necessary and sufficient condition for equality in (3.6) is that $a=b$ or $b=c$ or that $\phi(s)$ remains constant for both $a<s<b$ and $b<s<c$ (there can be a jump at $b$ ).

Proof of Lemma 3. Let $\sigma>0$ and $t_{0}$ be chosen arbitrarily. Let $j$ and $k$ be defined by $f_{t_{0}}(\sigma)=\xi_{j+1}\left(t_{0}\right)=\ldots=\xi_{j+k}\left(t_{0}\right)$ and $\xi_{j}\left(t_{0}\right)<\xi_{j+1}\left(t_{0}\right), \quad \xi_{j+k}\left(t_{0}\right)<\xi_{j+k+1}\left(t_{0}\right)$. Then $M_{j}<\sigma \leqq M_{j+k}$. We apply inequality (3.6) to $a=M_{j}, b=\sigma, c=M_{j+k}, \phi(s)=f_{t}(s)$, $\Phi(\sigma)=F_{t}(\sigma)$. Thus

$$
F_{t}(\sigma) \leqq \frac{M_{j+k}-\sigma}{M_{j+k}-M_{j}} F_{t}\left(M_{j}\right)+\frac{\sigma-M_{j}}{M_{j+k}-M_{j}} F_{t}\left(M_{j+k}\right) .
$$

This holds for $t$ in some neighborhoods of $t_{0}$, namely under the condition that the strict inequalities $\xi_{j}(t)<\xi_{j+1}(t)$ and $\xi_{j+k}(t)<\xi_{j+k+1}(t)$ still hold. On the right hand side of (3.7) we see the convex combination of two functions of $t$ which are concave on the interval considered, moreover equality holds for $t=t_{0}$ because $f_{t_{0}}(s)$ remains a constant for $M_{j}<s<M_{j+k}$. Thus $F_{t}(\sigma)$ is concave with respect to the variation of $t$. Q.E.D.

Lemma 4. Suppose $x(t)$ is a motion having the PCMC property and asymptotic velocities zero. Then $\lim _{t \rightarrow+\infty} F_{t}(\sigma)$ exist for all $\sigma>0$.

Proof. Suppose that $\xi_{1}\left(t_{0}\right)=\ldots=\xi_{j}\left(t_{0}\right)<\xi_{j+1}\left(t_{0}\right)$. Then $\xi_{1}(t) \leqq \ldots \leqq \xi_{j}(t)<\xi_{j+1}(t)$ for $t$ in some neighborhood of $t_{0}$. Hence $M_{j} \xi_{1}(t) \leqq \mu_{1} \xi_{1}(t)+\ldots+\mu_{j} \xi_{j}(t)$ $=F_{\mu, \xi(t)}\left(M_{j}\right)$. But this is a concave function of $t$ coinciding for $t=t_{0}$ with the left hand side. This shows that $\xi_{1}(t)$ is a concave function of $t$. We claim that $\xi_{1}(t)$ is 
also non-decreasing. For suppose the contrary, say $\xi_{1}(b)<\xi_{1}(a)$ for some $b>a$. Then if $t, t+\Delta t>b$ we have

$$
\frac{\xi_{1}(t+\Delta t)-\xi_{1}(t)}{\Delta t} \leqq \frac{\xi_{1}(b)-\xi_{1}(a)}{b-a}<0
$$

Now let $T_{1}, T_{2}, \ldots$ be an infinite sequence of intervals tending to $+\infty$ such that for some $j$ and all $k \xi_{1}(t)=x_{j}(t)$ for $t \in T_{k}$. Then the difference quotients

$$
\frac{x_{1}(t+\Delta t)-x_{1}(t)}{\Delta t}
$$

are bounded away from zero for $t$ in the $T_{k}$ and this makes $\lim _{t \rightarrow \infty} v_{1}(t)=0$ impossible, contrary to hypothesis. Thus $\xi_{1}(t)$ is concave non-decreasing. Similarly $\xi_{n}(t)$ is convex non-increasing. Now

$$
M_{n} \xi_{1}(t) \leqq F_{\mu, \xi(t)}(\sigma) \leqq M_{n} \xi_{n}(t)
$$

where $M_{n}$ is the total mass of the particles. This shows that as $t$ increases the value $F_{\mu, \xi(t)}(\sigma)$ remains inside a non-increasing family of intervals. For a concave function (see Lemma 3 ) this is only possible if it is non-decreasing. Thus it has a limit as $t \rightarrow+\infty$, proving Lemma 4 .

Let us partition the interval $(0, M]$, where $M=m_{1}+\ldots+m_{n}$, into smaller intervals $\Delta$ open on the low and closed on the high end, by considering all possible sums of the type $\sum_{j \in J} \mathrm{~m}_{j}$, where $J$ ranges through non-empty subsets of $\{1,2, \ldots, n\}$, and taking these numbers as the endpoints of the $\Delta$. There are only a finite number $<2^{n}$ of them; we denote the set of all the $\Delta$ by $D$. It is evident that if $\sigma<\sigma^{\prime}$ are both in $\Delta$ then $f(\sigma)=f\left(\sigma^{\prime}\right)$, independently of the $x_{1}, x_{2}, \ldots, x_{n}$ used in the definition, provided only that the $m_{1}, m_{2}, \ldots, m_{n}$ are fixed. Therefore, we may actually write $f(\sigma)=f(\Delta)$ (with a slight abuse of notation which should, however, not lead to misunderstanding).

Suppose now that we consider a motion $x_{1}(t), \ldots, x_{n}(t)$ satisfying the hypotheses of Lemma 4. We claim that then $\lim _{t \rightarrow+\infty} f_{t}(\Delta)$ exist for all $\Delta \in D$. Indeed, we can write

$$
f_{t}(\Delta)=\frac{F_{t}\left(\sigma^{\prime}\right)-F_{t}(\sigma)}{\sigma^{\prime}-\sigma}
$$

with $\sigma>\sigma^{\prime}$ both in $\Delta$, and use the conclusion of Lemma 4 .

Proof of Theorem 3. As explained at the beginning of Sect. 3, the asymptotic velocities are taken zero, and we examine the component $x_{1}(t), \ldots, x_{n}(t)$ of the motion along the arbitrarily chosen $x$-axis. We note that, for each $i$ and each instant of time $t, x_{i}(t)$ equals $f_{t}(\Delta)$ for some $\Delta \in D$. Let $S_{i}$ denote the set of numbers $y$ such that if $\varepsilon>0$ and $T$ are given arbitrarily then $t \geqq T$ exists with $\left|x_{i}(t)-y\right|<\varepsilon$. Every number in $S_{i}$ must be one of the limits $\lim _{t \rightarrow+\infty} f_{t}(\Delta)$, for if $y$ is not one of these then we can enclose $y$ in the interval $(y-\varepsilon, y+\varepsilon)$ so small that it contains none of 
those limits, in which case some $T$ exists for which $t \geqq T$ and $\Delta \in D$ implies $\left|f_{t}(\Delta)-y\right|>\varepsilon$ and so also $\left|x_{i}(t)-y\right|>\varepsilon$. Clearly $\liminf _{t \rightarrow+\infty} x_{i}(t)$ and $\limsup _{t \rightarrow+\infty} x_{i}(t)$ belong to $S_{i}$. But the continuity of $x_{i}(t)$ implies that $S_{i}$ is a connected set, i.e., if $\alpha, \beta \in S_{i}$ then all numbers between $\alpha$ and $\beta$ also belong to $S_{i}$. But, as we just saw, $S_{i}$ is a finite set, and this shows that it contains only one number, $\lim \inf x_{i}(t)=\lim \sup x_{i}(t)$.

Q.E.D.

\section{References}

1. Galperin, G.A.: On systems of perfectly elastic balls moving in space or in a box. Dan. SSSR 245, 285 (1979)

2. Moser, J.: The scattering problem for some particle systems on the line. In: Lecture Notes in Mathematics, Vol. 597, p. 441. Berlin, Heidelberg, New York: Springer 1977

3. Galperin, G.A.: Elastic collisions of particles on the line. Usp. Mat. Nauk. 33, 211 (1978)

4. Sinai, Ya.G.: Billiard trajectories in a polyhedral angle. Usp. Mat. Nauk. 33, 231 (1978)

5. Sinai, Ya.G.: An introduction to ergodic theory. Princeton, NJ : Princeton University Press 1976

6. Himchenko, N.G., Sinai, Ya.G.: Concerning the description of reflectionless potentials. Rep. Math. Phys. (in press)

7. Galperin, G.A.: On systems of locally interacting and repelling particles moving in space. Trudy MMO 43 (1981)

8. Vaserstein, L.N.: On systems of particles with finite-range and/or repulsive interactions. Commun. Math. Phys. 69, 31 (1979)

Communicated by D. Ruelle

Received June 17, 1980; in revised form December 7, 1981 